\title{
Luxation of Eye Ball Following Trauma - A Rare Case Presentation
}

\author{
Thapa R \\ Tilganga Institute of Ophthalmology, Gaushala, Kathmandu, Nepal.
}

\section{ABSTRACT}

Luxation of the eye globe is a rare event but it carries a risk of threat to permanent vision loss if appropriate intervention is not initiated on time. Although a rare presentation following minor trauma, it is usually associated with other ocular conditions like shallow orbital socket, floppy eyelid syndrome, and exophthalmos. Prompt reduction results in restoration of full anatomical and visual recovery in otherwise healthy eyes. We herein report a case of luxation of the eye ball following trauma in a 15-year-old girl, who recovered completely after the surgrical correction and a review of the relevant literature.

\section{INTRODUCTION}

Ocular traumas are among the common ocular morbidities leading to visual disability and blindness worldwide, including in Nepal. The Nepal Blindness Survey of 1981 estimated that trauma was responsible for $2.4 \%$ of total blindness and $7.9 \%$ of uniocular blindness. ${ }^{1}$ Luxation of the globe is a rare ocular condition following trauma where there is a forward displacement of the eye ball so that the eye lids spasmodically close behind it but the eye muscles and optic nerve generally remain intact unlike in avulsion of the globe where the muscles and optic nerve are partially or totally severed, usually because the eye has been knocked completely out of the socket. ${ }^{2}$ Although a rare ocular problem following trauma, there may be irreversible vision loss on delayed treatment. There are many case reports of globe luxation from other parts of world but to the best of my knowledge, no such cases have been reported from our country.

\section{CASE REPORT}

A fifteen-year-old girl from Rasuwa district of Nepal presented in the emergency room of Tilganaga Institute of ophthalmology, a tertiary eye care centre of Nepal, with the complain of protrusion of her left eye ball following trauma at the forehead and orbital region sustained when she had bumped into a door corner eight hours previously. Except blurring of vision and

\author{
Correspondence \\ Dr. Raba Thapa \\ Tilganga Institute of Ophthalmology \\ Gaushala, Kathmandu, Nepal. \\ Phone: 4493775 \\ Email:rabathapa@live.com
}


mild pain on that eye, there were no other ocular and systemic complains.

At presentation, her visual acuity on the right eye (RE) was $6 / 6$ and the left eye (LE) 2/60 with no improvement on best possible correction. The left ball was bulged out of the socket and both eyelid margins were turned in completely behind the eye ball. The conjunctiva was congested and chemosed (Figure 1).

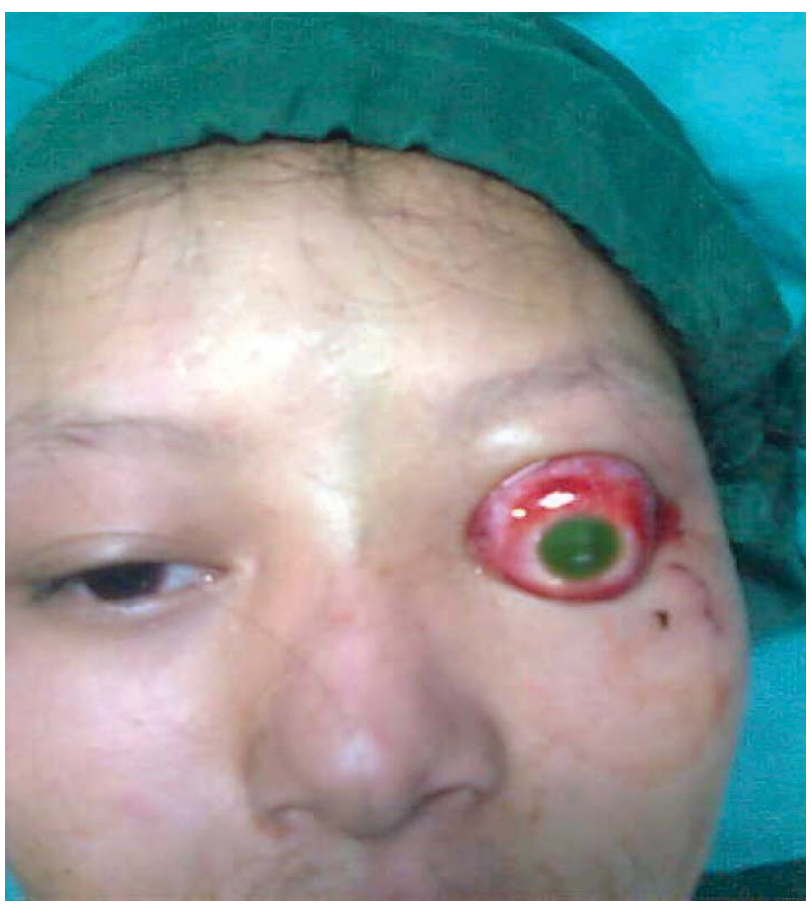

Figure 1. Pre-operative photograph with globe luxation

There was a large corneal abrasion measuring 7.5 $\mathrm{mm}$ and $10 \mathrm{~mm}$. The anterior chamber depth was normal and quiet. The pupil was $3 \mathrm{~mm}$, round, regular and reacting to light with the absence of any relative afferent papillary defect. The optic disc, macula and retinal periphery were grossly normal. The lids, adnexae and anterior and posterior segment findings on RE were unremarkable.

Under topical anesthesia, reduction of the globe was tried immediately but it was not successful. So, reduction of the globe was done under general anesthesia within two hours of presentation at the hospital by first making a lateral canthotomy and pulling the retracted eye lids out with gently pushing the globe back into the socket. There was a laceration on the upper lid, $4 \mathrm{~mm}$ above the lid margin, and it was repaired at the same setting and a temporary lateral tarsorrhapy was also done. Figure 2 shows the eye ball after reduction. The eye was padded with topical chloramphenicol ointment. The patient was prescribed for oral antibiotics (ampicillin and cloxacillin) $500 \mathrm{mg}$ four times a day for a week, oral Ibuprofen 400 $\mathrm{mg}$ and acetazolamide $250 \mathrm{mg}$ three times a day for three days each.

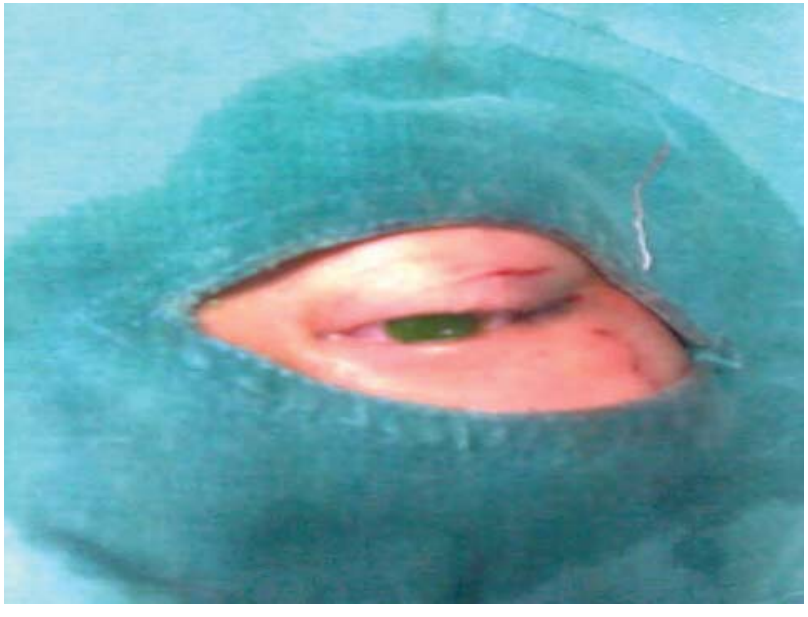

Figure 2. Post-operative photograph at three months follow-up.

During the first post-operative day, the patient was comfortable with a presenting visual acuity of $3 / 60$. The extra ocular movements were normal with the globe in its normal position. The size of the corneal epithelial defect decreased from $4 \mathrm{~mm}$ to $2 \mathrm{~mm}$. A small sphincter tear was noticed in the iris at the three $o$ 'clock position. The rest of the ocular findings were within normal limits.

The eye was again padded with chloramphenicol ointment besides the addition of oral prednisolone 35 mg under cover of a proton pump inhibitor. On the second post-operative day, visual acuity returned to $6 / 36$. The cornea was clear with no epithelial defect. The rest of the ocular findings were normal with the lateral tarsorrhapy in situ. The patient was advised for topical chloramphenicol eye drop 6 times a day and ointment three times a day.

A CT scan of the orbit showed a preseptal soft tissue thickening in the left orbit with an air pocket noted in the left extraconal space adjacent to the tendinous attachment of the left superior rectus muscle. The rest of the ocular findings on the extraocular muscles, retrobulbar space, globes, optic nerve sheath complex, bony orbit and orbital apex were normal.

On the seventh post-operative day, the visual acuity was $6 / 18$ on that eye. The lateral tarsorrhapy was removed. There was improvement of the conjunctival chemosis but a circumferential conjunctival laceration was noted at $6 \mathrm{~mm}$ posterior to the limbus and was repaired under local anesthesia. The intraocular pressure was normal $(16 \mathrm{~mm} \mathrm{Hg}$ ) on that eye. The patient was advised to continue oral prednisolone in a tapering dose and apply topical fluoromethalone one drop twice daily along with topical chloramphenicol.

During the $14^{\text {th }}$ post-operative day, the presenting visual acuity returned to $6 / 6$. There was significant improvement of the conjunctival chemosis. The rest 
of the anterior and posterior segment findings were unremarkable. Color vision was normal in both eyes. The patient was advised to continue topical fluoromethalone eye drop once a day for five days and topical antibiotics for another 10 days.

The visual acuity was 6/6 with unremarkable anterior and posterior segment findings at the subsequent two follow-ups at three months (Figure 3) and six months post-operatively. The patient was then advised to follow-up after three months or earlier as and when she felt it necessary.

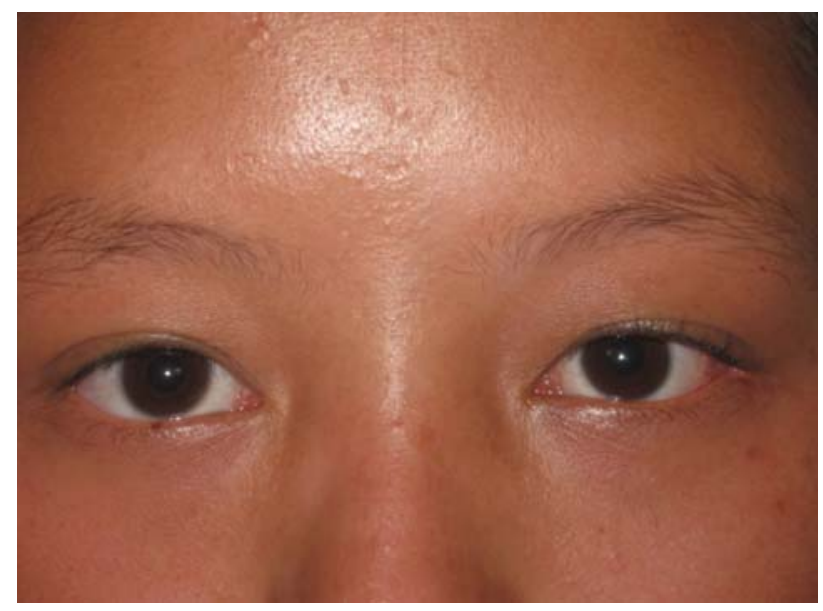

Figure 3. Post-operative photograph immediately following reduction of globe

\section{DISCUSSION}

Ocular morbidity following traumas is a common presentation leading to visual impairment and even uni or biocular blindness. Luxation of the globe is a rare presentation following minor trauma and usually occurs in patients with shallow orbital sockets, floppy eye-lid syndrome, and lax extraocular muscles. The risk factors include exophthalmos, which can be secondary to space-occupying orbital pathology such as excessive orbital fat, sarcoidosis, Graves' disease, or orbital tumors ${ }^{3}$.When an eye luxates, the globe quickly dries out leading to corneal abrasion and ulcer formation with further threatening the sight. Unlike the reported risk factors, our case had no such predisposing ocular conditions except the trauma sustained at the forehead and the orbit following a bump against a door corner.

The luxated globe should be managed as an emergency. Patient relaxation is a key factor in order to allow the orbiculais oculi muscle to relax and to make globe manipulation easier. The patient should be reclined, and sometimes the force of gravity is enough to allow the globe to slip back into the socket. The second step is rolling back the lids out around the front part of the globe using a finger of cotton swab while gently pushing the globe back into place. If severe blepharospasm, is present a local injection with lidocaine into the facial nerve is helpful. In conditions when reduction is not possible with this maneuver, it is easier to reduce it under sedation and with the help of a lateral canthotomy, as in our case.

Many cases with luxation of the eye ball associated with different ocular insults have been reported from other countries. The ocular injuries could be variable, from minor orbital insults to severe injuries with involvement of the globe. Van Der Wal and Van Der $\mathrm{Pol}^{4}$ have reported luxation of the eye ball in a 30 - yearold male following a minor orbital trauma. Zengin et $\mathrm{al}^{5}$ have reported another case of globe luxation following a trivial trauma. The luxation was accompanied by laceration of the conjunctiva as in our case and full functional recovery was possible following reposition of the globe. Likewise, Chhabra and Kauwma ${ }^{6}$ have reported luxation of the eye ball in two African patients following minor trauma to the face, and both were reduced without any permanent visual disability.

Lee et $\mathrm{al}^{2}$ reported a globe luxation following trauma associated with conjunctival laceration and extraocular muscle limitation in all directions. Orbital CT yielded proptosis and globe luxation without avulsion of the internal and external rectus muscles and the optic nerve. After a lateral canthotomy, they successfully treated the condition along with corticosteroid therapy, without visual impairment or eye ball movement disorder.

Kiratli et $\mathrm{al}^{7}$ reported the luxation of the globe associated with LeFort type III fractures and avulsion of the optic nerve and all extraocular muscles except for the medial rectus. Although the insulted eye had no light perception, most of its motility was restored resulting in an unblemished cosmesis.

Kunesh and $\mathrm{Katz}^{3}$ reported a case of spontaneous globe luxation which occurred with attempted contact lens placement and of successful reduction with no visual sequelae. Likewise, there are some reported cases with luxation of the eye ball in conditions like sarcoidosis, hyperemesis gravidarum, and Crouzon's disease with complete recovery following reduction of the globe and with a permanent tarsorrhaphy in Crouzon's disease, without any sequelae ${ }^{8,10}$.

Luxation of the globe is a rare presentation usually associated with other orbital anomalies, but a trivial ocular insult may lead to luxation of the globe even in the normal eyes and orbit. Complete anatomical and visual recovery results with prompt reduction in absence of other sight threatening ocular pathology. 


\section{REFERENCES}

1. Brilliant LB, Pokhrel RP, Grasset NC et al. Epidemiology of blindness in Nepal. Bull World Health Organ. 1985;63:37586.

2. Lee DY, Kwak HW, Kim SW. A Case of Traumatic Globe Luxation. J Korean Ophtalmol Soc. 2003;44(10):2434-8.

3. Kunesh JC, Katz SE. Spontaneous Globe Luxation Associated with Contact Lens Placement. CLAO Journal. 2002;28(1):2-4.

4. Van der Wal KG, Van der Pol BA. Traumatic luxation of the eyeball. J Craniomaxillofac Surg. 1991;19(5):205-7.

5. Zengin N, Karakurt A, Gültan E and Kural G. Traumatic globe luxation. Acta Ophthalmologica. 1992;70:844-6.
6. Chhabra HN and Kawuma AM. Luxation of the eyeball. Br J Ophthalmol. 1986;70(2):150-1.

7. Kiratli H, Tumer B, Bilgic S. Management of traumatic luxation of the globe. Acta Ophthalmol Scand. 1999;77(3):3402.

8. Lockshin NA, Hash KS, Cowen EW. Luxation of the eye ball. Journal of the American Academy of Dermatology. 2004;51(5):840

9. Zeller J, Murray SB, Fisher J. Spontaneous Globe Subluxation in a Patient with Hyperemesis Gravidarum: A Case Report and Review of the Literature. The Journal of Emergency Medicine. 2007;32(3):285-7.

10. Lim L, Wong PK. Luxation of the globe and Crouzon's disease--the first reported case in Singapore. Singapore Med J. 1996;37(5):553-5. 